\title{
Graphene Oxide Film as a Passive Q-switcher in an Erbium-doped Fiber Laser Cavity
}

\author{
Fauziah Che Mat ${ }^{1,2}$, Moh Yasin ${ }^{3}$, Anas Abdul Latiff ${ }^{4 *}$ and Sulaiman Wadi Harun ${ }^{2}$ \\ ${ }^{1}$ Politeknik Sultan Azlan Shah, 35950 Behrang, Perak, Malaysia \\ ${ }^{2}$ Photonics Engineering Laboratory, Department of Electrical Engineering, University of Malaya, 50603 Kuala \\ Lumpur, Malaysia \\ ${ }^{3}$ Department of Physics, Faculty of Science and Technology, Airlangga University, Surabaya, Indonesia \\ ${ }^{4}$ Centre of Telecommunication Research \& Innovation, Universiti Teknikal Malaysia Melaka, 76100 Hang \\ Tuah Jaya, Melaka, Malaysia
}

Received July 27, 2017; accepted September 28, 2017; published September 30, 2017

\begin{abstract}
All-fiber passively Q-switched fiber lasers have been demonstrated by using a graphene oxide (GO) Q-switcher for possible applications in telecommunication, laser processing, fiber sensing and medicine. The GO material was obtained through a modified Hummers method from expanded acid washed graphite flakes and was embedded into a polyvinyl alcohol (PVA) film to form a saturable absorber (SA) device. The Q-switched pulse operates at $1563.3 \mathrm{~nm}$ with a repetition rate that can be tuned from $44.33 \mathrm{kHz}$ to $61.77 \mathrm{kHz}$ as the pump power changes from $39 \mathrm{~mW}$ to $96 \mathrm{~mW}$. The highest repetition rate of $61.77 \mathrm{kHz}$ is achieved at a pump power of $96 \mathrm{~mW}$ and it is observed that the Qswitched pulse produced a maximum pulse energy of $0.054 \mathrm{~nJ}$ and pulse width of $5.57 \mu$ s at $96 \mathrm{~mW}$ pump power.
\end{abstract}

Q-switched fiber lasers are gaining great interest for various applications such as medicine, remote sensing, marking and machining. They can be realized using either active or passive techniques [1,2]. Compared with the active method, the passive Q-switching owns the unique advantage of a simple structure in all-fiber designing. The passive Q-switched laser can be realized by adopting a saturable absorber (SA) in the cavity. So far, many kinds of SAs have been reported, such as semiconductor saturable absorber mirrors (SESAMs) [2], carbon nanotubes (CNTs) [3, 4] and graphene [5]. SESAM has a narrow wavelength tuning range, and its modulation depth is typically low [2]. The CNTs and graphene are preferable SAs specifically for Q-switching operations because of their advantages including low saturation intensity, low cost and broadband wavelength operation [5].

Graphene is a preferable SA to replace the SESAMs. However, the preparation of a high quality graphene film is more difficult and expensive. Furthermore, graphene cannot be dissolved in water so that the efficiency for film fabrication by a graphene aqueous solution is decreased. Graphene oxide (GO) has traditionally served as a precursor for graphene because of its simple fabrication method and low cost $[6,7]$. In this paper, we demonstrate

\footnotetext{
*E-mail: anasabdullatiff@utem.edu.my
}

a Q-switched Erbium-doped fiber laser (EDFL) using a new GO material as SA. The SA device is fabricated by embedding a GO material into a polyvinyl alcohol (PVA) film. The GO material was obtained from expanded acid washed graphite flakes via a modified Hummers method. The proposed SA can be operated in a broad wavelength range because of its unselective absorption.

The GO was synthesized through a modified Hummers method from expanded acid washed graphite flakes, which involves many steps. First, a small amount of graphite powder $(5 \mathrm{~g})$ was poured into $125 \mathrm{ml}$ of $\mathrm{H}_{2} \mathrm{SO}_{4}$. Next, $2.75 \mathrm{~g} \mathrm{NaNO}_{3}$ reagents was added into the beaker to start the reaction. The mixture was kept into an ice water bath in order to keep it below $5^{\circ} \mathrm{C}$. A small amount of $\mathrm{KMnO}_{4}$ oxidant $(15 \mathrm{~g})$ was added in portions into the mixture under continuous stirring. After the addition of the $\mathrm{KMnO}_{4}$, the beaker was heated and kept at a temperature of around $30^{\circ} \mathrm{C}$ by continuously stirring the mixture. After that, the mixture was left at room temperature for more than 12 hours.

In the next stage, deionized water was added and the beaker was put into a water bath and stirred to maintain the temperature below $35^{\circ} \mathrm{C}$ for some time. Then, the mixture was heated to $95^{\circ} \mathrm{C}$ and kept under the same temperature for $15 \mathrm{~min}$ to initiate a reaction. Deionized water $(280 \mathrm{ml})$ and $\mathrm{H}_{2} \mathrm{O}_{2}(5 \mathrm{ml})$ were then added to stop the reaction. The mixture was rinsed with an $\mathrm{HCl}$ solution and deionized water to remove sulfate and chloride ions, respectively.

The GO solution was prepared by adding GO into DI water at a volume ratio of 1:10 and $1 \mathrm{ml}$ of polysorbate 80 solution as a surfactant. Then, the mixed solution was stirred for 2 hours until it became homogenous, followed by an hour of ultra-sonication. The host polymer was prepared by dissolving $1 \mathrm{~g}$ of polyvinyl alcohol powder [PVA, 40000MW, Sigma Aldrich] into $120 \mathrm{ml}$ of DI water and stirred until completely dissolved. To fabricate the $\mathrm{SA}$, the GO solution was mixed with a PVA suspension 
and followed by two-hour ultra-sonication. Then, the mixture was carefully poured into a petri dish to avoid any bubble and left dry at room temperature. After drying, the thin film was slowly peeled out. It was then cut into a small piece to attach into an FC/PC fiber ferrule. The ferrule was then matched with another fresh ferrule via a fiber adaptor after depositing a small amount of index matching gel onto the fiber end to construct an all-fiber SA device.

The SA device was integrated into an EDFL cavity for the Q-switching experiment as illustrated in Fig. 1. The inset figure shows the thin GO film. The laser cavity consists of a $2.4 \mathrm{~m}$ long erbium doped fibre (EDF) as an active medium, a wavelength division multiplexer (WDM), an isolator, the fabricated GO PVA SA and an 80/20 output coupler. A 980nm pump was launched into the EDF via WDM. The EDF with an Erbium ion absorption of $23 \mathrm{~dB} / \mathrm{m}$ at $980 \mathrm{~nm}$ was used. A polarization independent isolator was used to ensure unidirectional propagation of the oscillating laser in the ring laser cavity. The laser output was tapped out using a 80:20 coupler which keeps $80 \%$ of the light to oscillate in the ring cavity. The spectral characteristic was measured using an optical spectrum analyzer (OSA). The temporal characteristics were measured using a $500 \mathrm{MHz}$ oscilloscope and a $7.8 \mathrm{GHz}$ radio-frequency $(\mathrm{RF})$ spectrum analyser via a $1.2 \mathrm{GHz}$ photodetector.

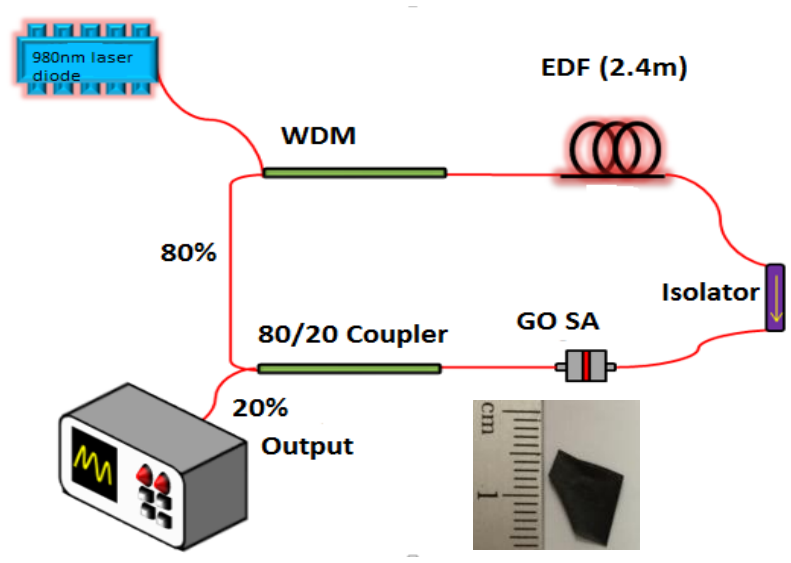

Fig. 1. Configuration of the GO PVA film based Q-switched EDFL. Inset shows the image of a GO film, which was sandwiched between two ferrules to form an SA device.

The stable Q-switching laser was self-started just by increasing the pump power over $39 \mathrm{~mW}$. There was no lasing below this pump power. Such a low threshold was most probably due to a small intra-cavity loss of the GO SA. A train of stable pulses with an increasing repetition rate was obtained as the pump power varied within 39 to $96 \mathrm{~mW}$. This is a typical characteristic for the Q-switched laser. Figure 2(a) shows the output spectrum of the EDFL at a threshold pump power of $39 \mathrm{~mW}$. As shown in the figure, the laser operated at a center wavelength of around $1563.3 \mathrm{~nm}$. Spectral broadening was also observed in the spectrum due to the self-phase modulation (SPM) effect. Figure 2(b) shows a typical oscilloscope trace of the Qswitched pulse train at a pump power of $96 \mathrm{~mW}$. It shows the peak to peak duration or a pulse period of $16.9 \mu \mathrm{s}$, which is equal to the repetition rate of $61.77 \mathrm{kHz}$. The pulse width is measured to be around $10.62 \mu \mathrm{s}$. The Qswitched pulse output was stable with no amplitude modulations in the pulses train. This indicates that there was no self-mode locking effect during the Q-switching operation. The film was then removed from the ring cavity for verifying that the passive Q-switching obtained was attributed to the GO SA device. We observed no Qswitched pulses on the oscilloscope trace even though the pump power was adjusted over a broad range. This finding further confirmed that the GO was fully responsible for the Q-switching operation of the EDFL.
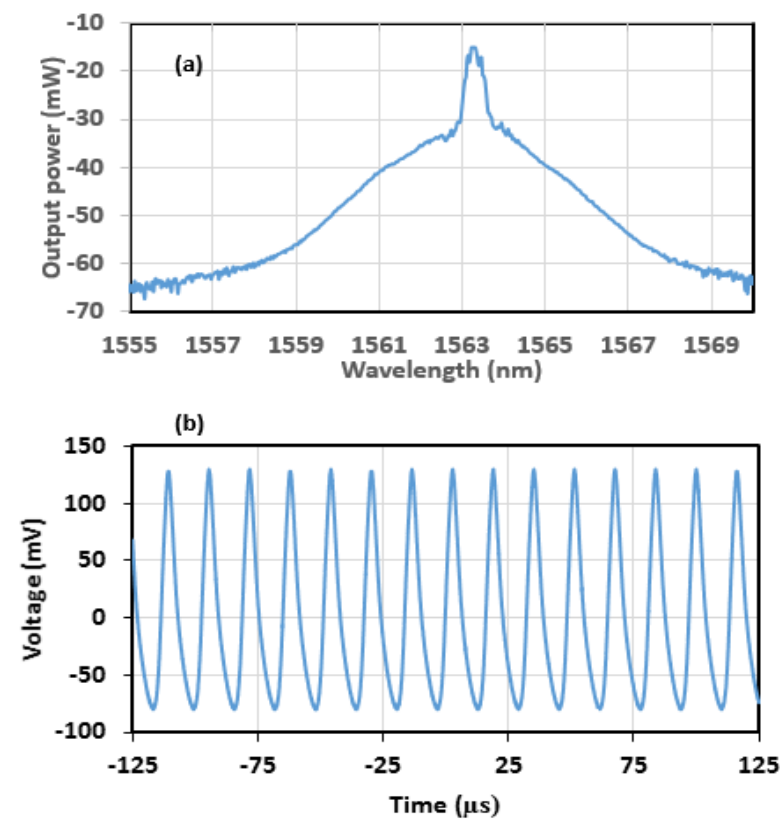

Fig. 2. Spectral and temporal characteristics: (a) output spectrum at $39 \mathrm{~mW}$ pump power; (b) typical pulses train at $96 \mathrm{~mW}$ pump power.

Figure 3(a) illustrates the relationship between the pulse repetition rate and pulse width with the pump power. When the pump power is varied from $39 \mathrm{~mW}$ to $96 \mathrm{~mW}$, the repetition rate increases almost linearly from $44.33 \mathrm{kHz}$ to $61.77 \mathrm{kHz}$. This is attributed that more gain is provided to saturate the SA as the pump power increases, which resulted in an increase of repetition rate. In contrast, pulse duration decreases from $9.51 \mu \mathrm{s}$ to $5.57 \mu \mathrm{s}$ as the pump power increases. We observe a smaller change of pulse width with the pump power at higher pump power. This is attributed to the fact that the SA is 
becoming saturated when more photons circulate inside the laser cavity as the pump power increased. The minimum attainable pulse duration is $5.57 \mu \mathrm{s}$, which is believed to be related to the modulation depth of the SA. The pulse duration can be further decreased by shortening the cavity length and improving the modulation depth of the SA.

Figure 3(b) shows the relationship between the average output power and pulse energy with the pump power. It is observed that both the average power and pulse energy increase with the increment of pump power. The average output power can be linearly increased from $1.3 \mu \mathrm{W}$ to $3.34 \mu \mathrm{W}$ by tuning the pump power from $39 \mathrm{~mW}$ to $96 \mathrm{~mW}$. The maximum pulse energy of $0.054 \mathrm{~nJ}$ was obtained at a pump power of $96 \mathrm{~mW}$. The increment of $980 \mathrm{~nm}$ pump power increases the average output power and reduces the pulse width, which in turn resulted in higher pulse energy to be extracted in the Q-switching process.
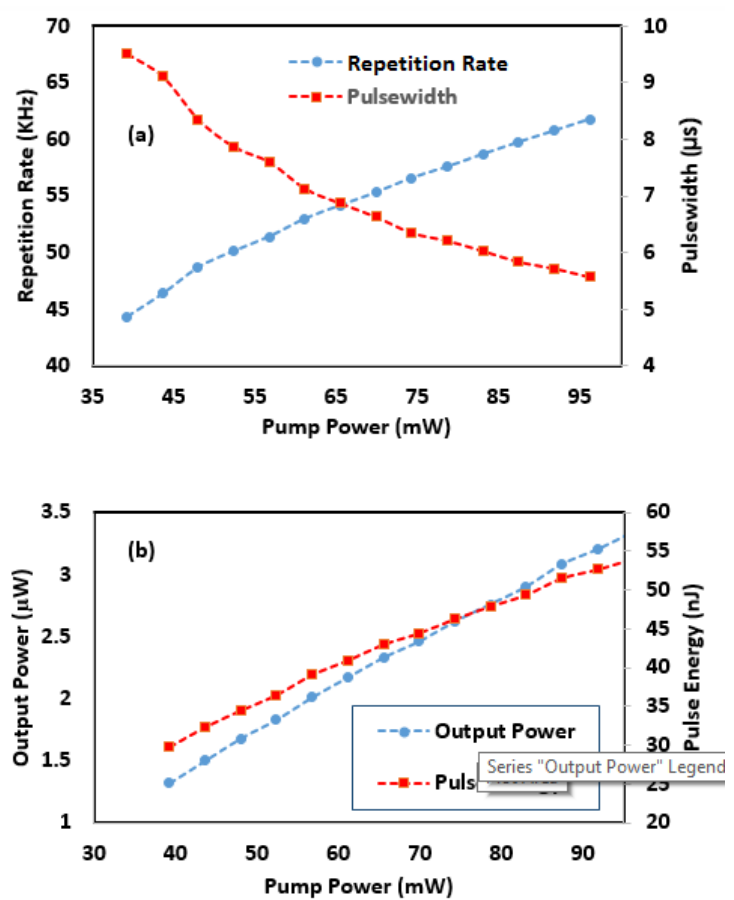

Fig. 3. Q-switching performances: (a) Repetition rate and pulse width as a function of pump power; (b) output power and pulse energy as a function of pump power.

To confirm the Q-switching stability, the radiofrequency $(\mathrm{RF})$ spectrum is also investigated at a pump power of $96 \mathrm{~mW}$. Figure 4 shows the RF spectrum, which indicates the fundamental frequency of $61.77 \mathrm{kHz}$ and a high signal to noise ratio (SNR) of $60 \mathrm{~dB}$. The SNR indicates good pulse train stability, comparable to other Q-switched fiber lasers based on CNT and graphene [6-8].
It is expected that a better Q-switched pulse can be obtained by optimizing the design of the cavity.

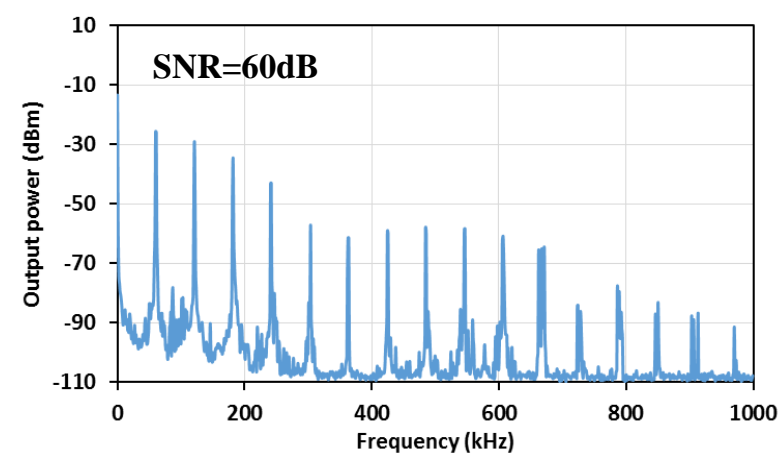

Fig. 4. RF spectrum at $96 \mathrm{~mW}$ pump power.

In conclusion, a passively Q-switched EDFL operating at $1563.3 \mathrm{~nm}$ was successfully demonstrated based on GO PVA SA. The SA device is fabricated by embedding a GO material into a PVA film. Employing this device into an EDFL cavity, we have achieved stable Q-switched pulses generation within a pump power range of 39 to $96 \mathrm{~mW}$. Through fine increasing the pump power, the repetition rate could be changed from $44.33 \mathrm{kHz}$ to $61.77 \mathrm{kHz}$, and the pulse duration from $9.51 \mu \mathrm{s}$ to $5.57 \mu \mathrm{s}$. The pulse energy was $0.054 \mathrm{~nJ}$ at a pump power of $96 \mathrm{~mW}$. These results show that GO is a new potential SA material for pulsed laser applications.

\section{References}

[1] J. Zayhowski, C. Dill, Opt. Lett. 20, 716 (1995).

[2] C.-X. Gao, W. Zhao, Y.-S. Wang, S.-L. Zhu, G.-F. Chen, Y.-G. Wang, 27th International Congress on High-Speed Photography and Photonics, 62794G (2007).

[3] M. Ahmed, N. Ali, Z. Salleh, A. Rahman, S. Harun, M. Manaf, Optics Laser Techn. 65, 25 (2015).

[4] S. Harun, M. Ismail, F. Ahmad, M. Ismail, R. Nor, N. Zulkepely, et al., Chinese Phys. Lett. 29, 114202 (2012).

[5] A. Martinez, Z. Sun, Nat. Photon. 7, 842 (2013).

[6] J. Boguslawski, J. Sotor, G. Sobon, R. Kozinski, K. Librant, M. Aksienionek, Photon. Research 3, 119 (2015).

[7] H. Ahmad, F.D. Muhammad, M.Z. Zulkifli, S.W. Harun, Chinese Opt. Lett. 11, 071401 (2013). 